Rev. Adm. Saúde - Vol. 18, № 71, abr. - jun. 2018

http://dx.doi.org/10.23973/ras.71.111

ARTIGO DE ATUALIZAÇÃO

\title{
Padrões de terminologias nacionais para procedimentos e intervenções na saúde
}

Nationals terminologies standards for procedures and interventions in healthcare

\section{Daiane Aparecida Maciel ${ }^{1}$, Deborah Pimenta Ferreira ${ }^{2}$, Heimar de Fátima Marin ${ }^{3}$}

1. Tecnóloga em processamento de dados. Mestranda do Programa de Pós-graduação em Gestão e Informática em Saúde da UNIFESP, São Paulo SP

2. Médica, doutora em informática médica pela Faculdade de Medicina da USP. Servidora da Secretaria Municipal da Saúde. São Paulo SP

3. Enfermeira, doutora em ciências biológicas. Professora titular e orientadora do Programa de Gestão e Informática em Saúde da UNIFESP. São Paulo SP

\section{RESUMO}

Objetivo: apresentar terminologias para registro de procedimentos e intervenções de saúde. Método: trata-se de artigo de atualização que relata informações atuais sobre o tema. Utilizou-se as bases de dados Scientific Eletronic Library Online (SCIELO), PUBMED e páginas eletrônicas da Agência Nacional de Saúde Suplementar (ANS), Ministério da Saúde (MS) e Associação Médica Brasileira (AMB). O período de busca contemplou estudos publicados nos últimos quinze anos em língua portuguesa. Resultado: das terminologias existentes, decidiu-se restringir o relato de atualização da Terminologia Unificada da Saúde Suplementar (TUSS), Classificação Brasileira Hierarquizada de Procedimentos Médicos (CBHPM), Tabela de Honorários Médicos (THM), Lista de Procedimentos Médicos (LPM), Tabela de Procedimentos, Medicamentos, Órteses, Próteses e Materiais Especiais do 
Sistema Único de Saúde (Tabela de Procedimentos do SUS) e SNOMED CT. Conclusão: Dada a importância de terminologias de saúde, é necessário investimento em recursos humanos, processo de governança e distribuição de terminologias.

Palavras-chaves: preços hospitalares; sistemas de informação em saúde; terminologia; interoperabilidade da informação em saúde.

\begin{abstract}
Objective: introduce terminologies for recording procedures and interventions in healthcare. Method: this is an update article which reports currents informations about the subject. Data bases Scientific Eletronic Library Online (SCIELO), PUBMED and eletronics pages of the National Supplementary Health Agency (ANS), the Ministry of Health (MS), and the Brazilian Medical Association (AMB) were used. The search period included studies published in the last fifteen years, in Portuguese. Results: from existing terminologies, it has been decided to restrict the update report of the Unified Health Terminology Supplementary (TUSS), Hierarchical Brazilian Classification of Procedures Doctors (CBHPM), Medical Fees Table (THM), List of Procedures Physicians (LPM), Management System of the Table of Procedures, Drugs and Orthoses, Prosthetics and Materials of SUS (Tabela de Procedimentos do SUS) and SNOMED CT. Conclusion: Given the importance of terminologies in healthcare, the investment in human resources, governance process and distribution of terminologies are necessary.
\end{abstract}

Keywords: hospital charges; health information systems; terminology; health information interoperability.

\title{
INTRODUÇÃO
}

O prontuário do paciente é um importante meio de comunicação não somente para viabilizar e dar continuidade ao atendimento, mas para servir de recurso para pesquisas e elaboração de novas evidências que possam incrementar os modelos de atendimento e gestão em saúde. Para tanto, é preciso que haja legibilidade, clareza, consistência e representatividade do dado e da informação do paciente. Neste sentido, o uso de padrões de terminologia em saúde tornou-se essencial para recuperação e análise da informação. O desafio, porém, é cada vez mais presente, principalmente para os profissionais que realizam a documentação à beira-leito, considerando que o registro de termos clínicos deve atender critérios como validade, especificidade, recuperação dos dados, facilidade de comunicação, sendo simples de entender, fácil de codificar e intuitivo aos profissionais de saúde (1). 
Também com a exponencial adoção da tecnologia da informação e comunicação em saúde e a promoção da interoperabilidade para melhorar a saúde, manter padrões consistentes e elementos de dados comuns são recursos estruturantes para o avanço dos modelos de atendimento na melhoria da qualidade e gestão. Esse avanço é baseado em objetivos como a captura de informações compartilháveis sobre pacientes e cuidados em todas as disciplinas e ambientes de atendimento, permitindo uma medição mais precisa e menos onerosa da qualidade do atendimento prestado e apoiando pesquisas e análises em andamento ${ }^{(2)}$.

Ainda, um dos principais desafios na troca de informações e uso de dados em qualquer setor de atendimento em saúde é a representação do dado e o uso nos registros eletrônicos. Embora o início do desenvolvimento dos primeiros padrões tenha sido mais evidente por volta de 1970 com o objetivo de auxiliar no desenvolvimento dos sistemas de saúde, foi na década de 1980 que, com a evolução dos sistemas e das terminologias, organizações foram se estabelecendo na tentativa de criar modelos integrados e complementares. Posteriormente, tais organizações passaram a ter um modelo mais competitivo e hoje, muitos dos padrões existentes servem para o mesmo propósito, mas o fazem de forma diversa na tentativa de se manter no mercado e atuar para incorporação nos registros eletrônicos e repositórios nacionais e mundiais de terminologias clínicas ou em saúde (SDOs - Standards Developing Organizations) ${ }^{(3)}$.

No Brasil são utilizadas as terminologias como a Classificação Estatística Internacional de Doenças e Problemas Relacionados à Saúde (CID), a CBHPM, a Tabela de Procedimentos do SUS, a Classificação Internacional de Atenção Primária (CIAP), e a TUSS. Estas terminologias e outras como SNOMED CT e a Terminologia Nomes e Códigos para Identificação de Observações Clínicas e Laboratoriais (LOINC) foram incluídas na portaria 2073/2011, que regulamenta o uso de padrões de interoperabilidade e informação em saúde, no âmbito do Sistema Único de Saúde (SUS) e nas esferas: municipal, distrital, estadual e federal, para os sistemas privados e do setor de saúde suplementar ${ }^{(4)}$. Esta portaria representou um grande avanço no campo normativo da avaliação e disponibilização da informação de estratégia em saúde, sua implementação promoverá um grande e contínuo esforço nacional. No desenvolvimento e uso de terminologias e implantação de registros eletrônicos, um dos termos mais utilizados tem sido "interoperabilidade", que se tornou muito importante pela necessidade de troca de registros de dados entre múltiplos sistemas e locais ${ }^{(3)}$. A interoperabilidade também é compreendida como a habilidade de comunicação ou troca de informação sobre entidades e relações entre diferentes termos, e o que permite a interoperabilidade entre os termos é a identificação de correspondências na qual possibilita o estabelecimento de uma linguagem unificada ${ }^{(5)}$.

Para atender a todos estes critérios, utiliza-se a terminologia, definida como representação estruturada de conceitos, legível por humanos e sistemas computacionais, possibilitando a uniformização nos campos dos serviços de saúde que facilita a interoperabilidade entre os mesmos, principalmente na assistência médico hospitalar ${ }^{(6)}$. 
Dessa forma, no seguimento de terminologias, é essencial acompanhar sua evolução, e, também, a melhor forma de implementação e aplicação dentro dos padrões de interoperabilidade para que se possa atingir o objetivo de integração e registros, preservando a segurança e privacidade dos dados dos pacientes e cidadãos.

O Brasil, como outros tantos países, utiliza tradicionalmente as terminologias e vocabulários padronizados com finalidade de classificação, precificação e levantamentos de informações. Para representar o conhecimento clínico no Registro Eletrônico de Saúde (RES), é essencial a utilização de terminologias estruturadas como a SNOMED CT.

Vale destacar que SNOMED CT é uma terminologia clínica, sistematizada, hierarquizada, composta de conceitos, descrições e correlações. Portanto, tem sido considerada fundamental para registrar a informação clínica no cuidado ao paciente viabilizando a recuperação da informação para continuidade do cuidado e realização de novos estudos, auxiliando na análise de resultados. Trata-se de uma nomenclatura multiaxial incluindo sinais e sintomas, diagnósticos e procedimentos abrangendo a maior parte das necessidades de documentação/registro de saúde ${ }^{(7,8)}$.

$\mathrm{Na}$ intenção de identificar na literatura as terminologias mais utilizadas para representar os procedimentos e intervenções na área da saúde, justifica-se a elaboração deste artigo, no qual o foco será apresentar as atualizações presentes no desenvolvimento e uso de terminologias mais utilizadas em nossa prática local.

Espera-se que a atualização aqui apresentada possa ser útil para fornecer maior entendimento na representação dos procedimentos e intervenções na área de saúde, apoiando o profissional que utiliza as terminologias com informações sobre o conceito, tipos de cobrança, período de vigência e atualizações.

\section{MÉTODOS}

Trata-se de um artigo de atualização que utilizou as bases de dados, Scientific Eletronic Library Online (SCIELO), PUBMED; e páginas eletrônicas da Agência Nacional de Saúde Suplementar (ANS), Ministério da Saúde (MS), e Associação Médica Brasileira (AMB). O período de busca contemplou os estudos publicados nos últimos quinze anos, em língua portuguesa. Os descritores utilizados para a busca foram: tabelas de remuneração de serviços, sistemas de informação de saúde, terminologia, terminologia atualizada de procedimentos médicos.

\section{RESULTADOS}

Inicialmente, vale lembrar que um artigo de atualização aborda informações publicadas sobre tema de interesse para determinada especialidade ${ }^{(9)}$. Assim, 
o relato que se segue, descreve as terminologias mais utilizadas para o registro de procedimentos e intervenções na área de saúde, a saber: THM, LPM, CBHPM, Tabela de Procedimentos do SUS, TUSS.

Considerando a importância e os futuros direcionamentos do Ministério da Saúde, decidiu-se também incluir a SNOMED CT.

\section{Tabela de Honorários Médicos (THM)}

Em 1962, dado a necessidade de ter uma tabela de referência para pagamento, o Departamento Nacional de Previdência Social (DNPS) solicitou à Associação Paulista de Medicina (APM) valores para a tabela de honorários médicos, criando a primeira tabela de faturamento do DNPS ${ }^{(10)}$.

Em 1967, foi criada a primeira Tabela de Honorários Médicos (THM) pela Associação Médica Brasileira (AMB), sua precificação é baseada em Coeficiente de Honorários Médicos $(\mathrm{CH})$, seus procedimentos são distribuídos por especialidade médica, sendo atualizadas periodicamente por meio de sua Comissão de Honorários Médicos, disponibilizando as edições: 1970, 1974, 1976, 1978 e 1983.

Em 1984, foi publicada uma nova versão da THM contendo novo sistema de codificação, sendo atualizadas e disponibilizadas novas edições nos anos de 1987 a 1992. Esta terminologia foi utilizada para diversas finalidades pelas instituições de saúde, como classificar, precificar, faturar e levantar informações. Em agosto de 2003, por meio de comunicado oficial publicado no Diário Oficial da União, a AMB teve os seus trabalhos encerrados, passando a vigorar a CBHPM ${ }^{(11)}$.

\section{Lista de Procedimentos Médicos (LPM)}

A Lista de Procedimentos Médicos (LPM) foi criada em 1996 pela a AMB, diferente das tabelas anteriores, que são precificadas por meio de Coeficiente de Honorários Médicos $(\mathrm{CH})$, esta nova versão modificou a precificação, seus valores passaram a ser expressos em moeda real. Esta tabela foi atualizada em 1999, seus procedimentos são distribuídos por especialidade médica. Esta terminologia foi utilizada para diversas finalidades pelas instituições de saúde, como classificar, precificar e levantar informações. Em agosto de 2003, por meio de comunicado oficial da AMB teve os seus trabalhos encerrados, passando a vigorar a CBHPM (11).

\section{Classificação Brasileira Hierarquizada de Procedimentos Médicos (CBHPM)}

Dado à necessidade de atualização das tabelas de faturamento, a AMB, conjunto com as Sociedades de Especialidade, o Conselho Federal de 
Medicina e a Federação Nacional dos Médicos, em 2003, publicou a $1^{\text {a }}$ versão da tabela Classificação Brasileira Hierarquizada de Procedimentos Médicos (CBHPM). Nesta, foi adotado um novo sistema de codificação, estruturação, forma de precificação, e passou a ser ordenada por região anatômica, seus valores passaram a ser expressos em portes e uma nova coluna foi criada: custos operacionais ${ }^{(11)}$. Ao longo dos anos, foram disponibilizadas as versões CBHPM 3aㅡ edição, contendo 3.968 registros; CBHPM 4aㅡ edição, contendo 4.025 registros; CBHPM 5a edição, contendo 4.154 registros; CBHPM 2010, contendo 4.289 registros; CBHPM 2012, contendo 4.349 registros; CBHPM 2014, contendo 4.535 registros; a versão mais recente é a CBHPM 2016, contendo 4.535 registros. A cada lançamento de nova versão, tem-se observado, no conjunto da terminologia, o aumento do número de termos (12). Estas terminologias são utilizadas para diferentes finalidades, de acordo com a necessidade da instituição de saúde.

Do ponto de vista de governança, há registros que indicam fóruns de construção de consenso na área da saúde. Exemplos: AMB, as Sociedades de Especialidade, o Conselho Federal de Medicina (CFM) e a Federação Nacional dos Médicos. A AMB é responsável por manter e atualizar a terminologia CBHPM ${ }^{(12)}$.

\section{Tabela de Procedimentos, Medicamentos, Órteses, Próteses e Materiais Especiais do Sistema Único de Saúde (Tabela de Procedimentos do SUS)}

O SUS utiliza a Tabela de Procedimentos do SUS. Foi instituída pela Portaria GM n 321 de fevereiro de 2007 e publicada pela Portaria GM no 2848 de novembro de 2007. A Tabela de Procedimentos do SUS contempla precificação, relacionamentos com Classificação Estatística Internacional de Doenças e Problemas Relacionados com a Saúde (CID10), Classificação Brasileira de Ocupações (CBO) e especialidade médica. Essa terminologia é organizada por grupos, subgrupos e forma de organização, sua atualização é mensal e seu versionamento é por competência (mês/ano).

A forma de codificação na Tabela de Procedimentos do SUS permite a identificação do procedimento de forma mais direta. O código é composto por dez dígitos, sendo os dois primeiros correspondentes ao Grupo, o terceiro e quarto correspondem ao Subgrupo, quinto e sexto correspondem a Forma de Organização, os três próximos são números sequenciais dentro da Forma de Organização e o último é um dígito verificador.

Do ponto de vista de governança o Departamento de Informática do SUS (DATASUS) é responsável por manter a atualização desta terminologia, a partir das requisições do seu gestor de negócio, o Departamento de Regulação, Avaliação e Controle de Sistemas (DRAC) ${ }^{(13)}$. A cada mês, é disponibilizada uma nova competência, que pode ser denominada versionamento. É disponibilizado um arquivo no formato txt, e está disponível no site do MS (http://Tabela de Procedimentos do SUS.datasus.gov.br/tabelaunificada/app/sec/inicio.jsp). O formato txt é um arquivo de texto utilizado para armazenar informações. 


\section{Terminologia Unificada da Saúde Suplementar (TUSS)}

A Agência Nacional de Saúde Suplementar (ANS) é o órgão do Governo Federal que regula as Operadoras de planos de saúde no Brasil, em 2005, publicou o padrão TISS por meio da Resolução Normativa (RN) de no 114 que determina a troca de informações na saúde suplementar, tornando obrigatório sua adoção ${ }^{(14) .}$

Com a implantação do padrão TISS, a ANS identificou as diferentes terminologias praticadas pelo mercado da saúde suplementar, dificultando a análise dos dados na troca de informações entre os atores envolvidos (prestadores de serviços e operadoras de planos de saúde), o que determinou a criação, em 2009, da TUSS ${ }^{(15)}$.

O objetivo da TUSS é consolidar, em uma única tabela, terminologias diferentes, em sua primeira edição representa os termos para procedimentos e serviços auxiliares de diagnose e terapia (SADT), não é precificada, e foi baseada na tabela de faturamento CBHPM, versão 2005.

Do ponto de governança, a Associação Médica Brasileira (AMB) é a entidade autora e responsável pela elaboração da terminologia relacionada aos procedimentos médicos e ficará responsável pela indicação das inclusões, com seus respectivos códigos e descrições nos modelos estabelecidos, conforme a

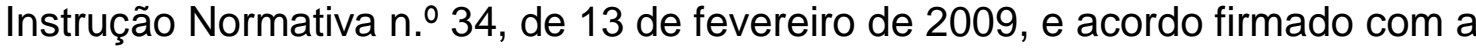
ANS.

O gerenciamento da TUSS é realizado de forma colegiada contando com a colaboração de inúmeras entidades setoriais de medicina, odontologia, enfermagem, medicamentos, órteses, próteses entre outros. Após dois anos da sua obrigatoriedade, esta terminologia também passou a compor diárias, taxas, gases medicinais, materiais, órteses, próteses, materiais especiais (OPME) e medicamentos. A solicitação de inclusão de novo código deverá ser feita por meio de formulário disponibilizado no sítio eletrônico da ANS, e encaminhado para análise. Cabe à ANS, por intermédio da Diretoria de Desenvolvimento Setorial (DIDES), disponibilizar os códigos e termos em seu sítio eletrônico, após aprovação do Comitê de Padronização das Informações em Saúde Suplementar (COPISS) e da ANS $\left({ }^{16}\right)$.

\section{SNOMED CT}

Em 2011, o MS publicou a portaria 2073/2011, que estabeleceu os o uso de padrões de informação em saúde e de interoperabilidade entre os sistemas de informação do SUS. No capítulo II, Catálogo de Padrões de Informação, estabelece o uso da terminologia SNOMED CT para representar codificação de termos clínicos e mapeamento das terminologias ${ }^{(4)}$.

A SNOMED CT resulta da unificação de duas terminologias: a SNOMED Reference Terminology - SNOMED RT desenvolvida pelo Colégio Americano 
de Patologistas (CAP) e a Clinical Terms Version 3 (CTV3) (conhecida como Read Codes) mantida pelo National Health Service - NHS do Reino Unido. Em acordo celebrado em 1999, as entidades possibilitaram a criação de uma terminologia única, SNOMED CT, que se tornou mais complexa com múltiplas possibilidades para codificar expressões.

A SNOMED CT permite relacionar diversos conceitos. Cada conceito codificado está ligado a diversos outros conceitos relacionados, tanto por meio de hierarquias multiaxiais (relações de tipo "é um"), quanto por definições lógicas (atributos) ${ }^{(17)}$. O uso deste formato de correlacionar hierarquias e lógicas possibilita maior expressividade, permitindo seu uso na codificação de informação da assistência individual ao paciente, na geração de informação adequada ao seu atendimento, na integração com diretrizes e protocolos clínicos, sistemas de apoio à decisão e o intercâmbio de informações entre diferentes atores do processo de assistência à saúde. Adicionalmente, os dados codificados, utilizando os formalismos da SNOMED CT, podem ser extraídos, gerando informações importantes para pesquisas e monitoramento de doenças.

A SNOMED CT consiste, portanto, em uma normatização global de terminologias clínicas, possuindo grande abrangência. Está traduzida em muitos idiomas, contando com amplo reconhecimento científico, permitindo representar o conhecimento clínico no RES de forma consistente.

\section{DISCUSSÃO}

As terminologias apresentadas demonstram a falta de padronização, fator essencial para a produção do conhecimento em saúde. O padrão Troca de Informação da Saúde Suplementar (TISS) por meio da Instrução Normativa no. 34 , de 13 de fevereiro de 2009, é uma iniciativa do governo para padronizar as terminologias ${ }^{(14)}$.

A adoção de terminologias e vocabulários controlados no ambiente da assistência à saúde no Brasil ultrapassa a mera disponibilização de tabelas, e foi reconhecida por meio da estratégia de e-Saúde para o Brasil (18).

No projeto de e-Saúde foi recomendado pelos especialistas a adesão ao SNOMED CT como terminologia de referência. O mapeamento existente do SNOMED CT para Classificação Internacional de Doenças 10 (CID10) está sendo adequado para garantir a aderência a Classificação Internacional de Doenças 11 (CID11) $^{(18)}$.

O atual quadro de uso de terminologias em saúde no Brasil, caracterizado pela diversidade de abordagens e ausência de padronização, demonstra a necessidade de aprofundar e consolidar os mecanismos de gestão de vocabulários e terminologias.

Um aspecto fundamental para o sucesso da adoção e consolidação do uso de terminologias controladas em saúde no Brasil, é a necessidade de superar o 
modelo de fornecimento de tabelas destituídas de conteúdo semântico e sem mecanismos formais de representação de conceitos. Essa característica é essencial para a garantia da interoperabilidade entre sistemas, ganhará impulso com a recente associação do Brasil ao SNOMED Internacional o que permitirá estruturar um modelo nacional de interoperabilidade baseado nas melhores práticas internacionais ${ }^{(18)}$.

O uso da SNOMED CT em sistemas de informação em saúde requer investimento, não é simples. A literatura sobre o assunto apresenta artigos que focam experiências isoladas, êxitos e dificuldades de implementação.

Um estudo contemplando 13 entrevistas com representantes de países que implementaram a SNOMED CT em sistemas de informação em saúde com entrevistados que participaram representando os Estados Unidos, Reino Unido, Canadá, Nova Zelândia e Austrália relataram suas experiências na fase de implantação. Os desafios reportados pelos entrevistados para o uso desta terminologia estão: falhas de cobertura de conteúdo da SNOMED CT, falhas nas relações hierárquicas da SNOMED CT, ambiguidade de termos da SNOMED CT, inconsistência sintática da SNOMED CT, ausência de tradução da SNOMED CT para outras línguas, incompreensão dos usos da póscoordenação da SNOMED CT, dificuldade de uso de subconjuntos da SNOMED CT, dificuldade de recuperação da informação via hierarquias da SNOMED CT ${ }^{(19) .}$

Esta atualização não tem a pretensão de discorrer ou apresentar sugestões para a adoção de terminologias controladas no Brasil. Entretanto, é evidente a atual utilização de terminologias e listas de termos por inúmeros atores da área de saúde.

A implantação de processos e práticas, metodologicamente estruturados, para o tratamento das terminologias em saúde exigirá o desenvolvimento de amplos programas de qualificação de recursos humanos. Um projeto de tal magnitude e complexidade exigirá, tanto dos poderes públicos como dos atores privados.

Esta é a tarefa que o Brasil tem pela frente para criar e consolidar um sistema de saúde interoperável.

\section{CONCLUSÃO}

Conclui-se que é essencial a adoção de terminologias estruturadas para representar o conhecimento clínico no RES. No momento em que o governo brasileiro estrutura uma estratégia de e-Saúde, que pretende disseminar a utilização de terminologias mais avançadas, como a SNOMED CT, torna-se necessário estabelecer um panorama geral da utilização dessas terminologias, investir em recursos humanos, processo de governança e distribuição de terminologias. 


\section{REFERÊNCIAS}

1. Marin HF. Terminologia de referência em enfermagem: a Norma ISO 18104. Acta Paul Enferm [Internet]. 2009 [cited 2018 Apr 29]; 22(4): 445-8.

Available from: http://www.scielo.br/pdf/ape/v22n4/a16v22n4.pdf

2. Healtit: The Office of the National Coordinator for Health Information Tecnhology. Standard Nursing Terminologies: a Landscape analysis. 2017 Mai [cited 2018 Jan 10]. Available from: https://www.healthit.gov/sites/default/files/snt final 05302017.pdf

3. Hammond EW. Standards for Global health information systems. In: Marin $\mathrm{H}$ F; Massad E, Gutierrez MA, Rodrigues RJ, Sigulem D. Global Health Informatics - how Information technology can change our lives in a globalized world. London: Elsevier. 2017, p. 94-108.

4. Ministério da Saúde (BR). Portaria no. 2.073 de 31 de agosto de 2011. Regulamenta o uso de padrões de interoperabilidade e informação em saúde para sistemas de informação em saúde no âmbito do Sistema Único de Saúde, nos níveis Municipal, Distrital, Estadual e Federal e para os sistemas privados e do setor de saúde suplementar. Diário Oficial da União: República Federativa do Brasil. 2011 Ago 31 [cited 2018 Jan 10]. Available from:

http://bvsms.saude.gov.br/bvs/saudelegis/gm/2011/prt2073_31_08_2011.ht $\mathrm{ml}$

5. Bittner T, Donnelly M, Winter S. Ontology and Semantic Interoperability. Institute for Formal Ontology and Medical Information Science (IFOMIS) Saarland University and Department for Geomatics The University of Melbourne. Available from:

https://pdfs.semanticscholar.org/890a/f1bd9b072bae84a7c3d67925bc0e8a0 4a68a.pdf

6. International Organization for Standardization. ISO/TR 12300:2014 [Internet]. Geneva: ISO, 2014 [cited 2018 Abr 15]. Available from: https://www.iso.org/standard/51344.html

7. Rasmussen AR, Rosenbeck K. SNOMED CT implementation: implications of choosing clinical findings or observable entities. Stud Health Technol Inform. 2011; 169:809-13.

8. Shahpori R, Doig C. Systematized Nomenclature of Medicine-Clinical Terms direction and its implications on critical care. J Crit Care. 2009:1-9.

9. Curty MG, Boccato VRC. O artigo Científico como forma de comunicação do conhecimento na área de Ciência da Informação. Perspecrt Ciênc Inf. 2005; 10(1):94-107.

10. Associação Médica Brasileira. Histórico das THMs, LPMs E CBHPMs. São Paulo: AMB, 2017. 
11. Associação Médica Brasileira. Comunicado Oficial. Diário Oficial da União. 13 fev 2004. Seção 3: 89. Disponível em:

https://www.jusbrasil.com.br/diarios/459190/pg-89-secao-3-diario-oficial-dauniao-dou-de-13-02-2004

12. Associação Médica Brasileira. Classificação Brasileira Hierarquizada de Procedimentos Médicos [Internet]. São Paulo: Manole, 2016 [cited 2018 Jan 30]. Available from: https://amb.org.br/ arquivos/ downloads/CBHPM2016.pdf.

13. Ministério da Saúde (BR). Sistema de Gerenciamento da Tabela de Procedimentos, Medicamentos e OPM do SUS [Internet]. 2018 [cited 2018 May 10]. Available from: http://sigtap.datasus.gov.br/tabelaunificada/app/sec/inicio.jsp

14. Agência Nacional de Saúde Suplementar (BR). Resolução Normativa no. 114 , de 26 de Outubro de 2005. Estabelece o padrão obrigatório para a troca de informações entre operadoras de plano privado de assistência à saúde e prestadores de serviços de saúde sobre os eventos de saúde, realizados em beneficiários de plano privado de assistência à saúde e dá outras providências [Internet]. Diário Oficial da União. 2005 Out 26 [cited 2018 Jan 10]. Available from:

http://www.ans.gov.br/component/legislacao/?view=legislacao\&task=TextoL ei\&format=raw\&id $=M T A w O Q==$

15. Agência Nacional de Saúde Suplementar (BR). Instrução Normativa no. 34, de 13 de Fevereiro de 2009. Dispõe sobre a instituição da Terminologia Unificada da Saúde Suplementar TUSS do Padrão TISS para procedimentos em saúde para a troca de informações entre operadoras de plano privado de assistência à saúde e prestadores de serviços de saúde sobre os eventos assistenciais realizados aos seus beneficiários [Internet]. Diário Oficial da União: República Federativa do Brasil. 2009 Fev 13 [cited 2018 Jan 10]. Available from:

http://www.ans.gov.br/component/legislacao/?view=legislacao\&task=TextoL ei\&format=raw\&id=MTM5NA==

16. Agência Nacional de Saúde Suplementar (BR). Operadoras e Serviços de Saúde: esclarecimento sobre Terminologia Unificada em Saúde Suplementar [Internet]. 2010 [cited 2018 May 10]. Available from: http://www.ans.gov.br/a-ans/sala-de-noticias-ans/operadoras-e-servicos-desaude/432-esclarecimento-sobre-terminologia-unificada-em-saudesuplementar

17. Ferreira DP; Lopes PRL. Padrões de Normatização em Informática em Saúde. São Paulo: UNIFESP. 2016.

18. Ministério da Saúde (BR). Estratégia de e-Saúde para o Brasil [Internet]. Brasília: MS, 2017 [cited 2018 Mai 06]. Available from: http://portalarquivos.saude.gov.br/images/pdf/2017/julho/12/Estrategia-esaude-para-o-Brasil.pdf 
19. Lee $D$, Cornet R, Laua F, Keizerb N. A survey of SNOMED CT implementations. Journal of Biomedical Informatics [Internet]. 2013 [cited 2018 May 13]; 46(1):87-96. Available from:

https://www.sciencedirect.com/science/article/pii/S1532046412001530

Recebido: 17 de maio de 2018. Publicado: 05 de junho de 2018

Correspondência: Daiane Aparecida Maciel. E-mail:

maciel.daiane@gmail.com

Conflito de Interesses: os autores declararam não haver conflito de interesses.

(C) This is an Open Access article distributed under the terms of the Creative Commons Attribution License, which permits unrestricted use, distribution, and reproduction in any medium, provided the original work is properly cited 